\title{
Long-Term Oral Methylphenidate Treatment in Adolescent and Adult Rats: Differential Effects on Brain Morphology and Function
}

\author{
Kajo van der Marel*,', Anne Klomp ${ }^{2}$, Gideon F Meerhoff ${ }^{3}$, Pieter Schipper ${ }^{4}$, Paul J Lucassen ${ }^{3}$, \\ Judith R Homberg ${ }^{4}$, Rick M Dijkhuizen ${ }^{1,5}$ and Liesbeth Reneman ${ }^{2,5}$ \\ 'Biomedical MR Imaging and Spectroscopy Group, Image Sciences Institute, University Medical Center Utrecht, Utrecht, The Netherlands; \\ ${ }^{2}$ Department of Radiology, Academic Medical Center, University of Amsterdam, Amsterdam, The Netherlands; ${ }^{3}$ Swammerdam Institute for Life \\ Sciences, Center for Neuroscience, University of Amsterdam, Amsterdam, The Netherlands; ${ }^{4}$ Department of Cognitive Neuroscience, Centre for \\ Neuroscience, Donders Institute for Brain, Cognition, and Behaviour, Radboud University Nijmegen Medical Centre, Nijmegen, The Netherlands
}

\begin{abstract}
Methylphenidate is a widely prescribed psychostimulant for treatment of attention deficit hyperactivity disorder (ADHD) in children and adolescents, which raises questions regarding its potential interference with the developing brain. In the present study, we investigated effects of 3 weeks oral methylphenidate $(5 \mathrm{mg} / \mathrm{kg})$ vs vehicle treatment on brain structure and function in adolescent (post-natal day $[P] 25)$ and adult (P65) rats. Following a I-week washout period, we used multimodal magnetic resonance imaging (MRI) to assess effects of age and treatment on independent component analysis-based functional connectivity (resting-state functional MRI), D-amphetamineinduced neural activation responses (pharmacological MRI), gray and white matter tissue volumes and cortical thickness (postmortem structural MRI), and white matter structural integrity (postmortem diffusion tensor imaging (DTI)). Many age-related differences were found, including cortical thinning, white matter development, larger dopamine-mediated activation responses and increased striatal functional connectivity. Methylphenidate reduced anterior cingulate cortical network strength in both adolescents and adults. In contrast to clinical observations from ADHD patient studies, methylphenidate did not increase white matter tissue volume or cortical thickness in rat. Nevertheless, DTI-based fractional anisotropy was higher in the anterior part of the corpus callosum following adolescent treatment. Furthermore, methylphenidate differentially affected adolescents and adults as evidenced by reduced striatal volume and myelination upon adolescent treatment, although we did not observe adverse treatment effects on striatal functional activity. Our findings of small but significant age-dependent effects of psychostimulant treatment in the striatum of healthy rats highlights the importance of further research in children and adolescents that are exposed to methylphenidate.

Neuropsychopharmacology (2014) 39, 263-273; doi:10.1038/npp.2013.169; published online 9 October 2013
\end{abstract}

Keywords: development/developmental disorders; clinical or preclinical; methylphenidate; attention deficit hyperactivity disorder; MRl; rats

\section{INTRODUCTION}

Attention deficit hyperactivity disorder (ADHD) is a highly prevalent neurodevelopmental disorder among children and adolescents (Pastor and Reuben, 2008). Although psychostimulants such as methylphenidate provide effective pharmacological treatment, core symptoms return on termination of treatment (Jensen et al, 2007). Despite its widespread prescription, little is known about the long-term effects of stimulant medication. To increase our understanding of psychostimulant interaction with the developing

*Correspondence: Dr K van der Marel, Biomedical MR Imaging and Spectroscopy Group, Image Sciences Institute, University Medical Center Utrecht, Yalelaan 2, 3584 CM Utrecht, The Netherlands, Tel: +31 30 2535568, Fax: +31 30 253556I, E-mail: kajo@invivonmr.uu.nl ${ }^{5}$ These authors contributed equally to this work.

Received 5 December 2012; revised 6 July 20 I3; accepted 8 July 20 I3; accepted article preview online 15 July 2013 brain, and to improve the effect of pharmacological intervention, recent research has focused on the neurobiological underpinnings of the disorder (Ashtari et al, 2005; Carmona et al, 2005; Giedd et al, 1994; Shaw et al, 2007), and effects of psychostimulant medication (Castellanos et al, 2002; Semrud-Clikeman et al, 2006; Shaw et al, 2009; Silk et al, 2009).

ADHD patients who did not receive psychostimulant medication were found to have smaller total gray and white matter volumes as compared with controls (Carmona et al, 2005; Castellanos et al, 2002). In these retrospective studies, psychostimulant medication did not affect gray matter volume, but overall white matter volume appeared normalized after treatment (Castellanos et al, 2002). Similarly, childhood psychostimulant medication was found to normalize ADHD-related reductions of anterior cingulate cortex volume, whereas caudate nuclei volumes remained markedly reduced regardless of medication 
history (Semrud-Clikeman et al, 2006). Yet, the shapes of the caudate nuclei of untreated patients showed inward deformations that appeared normalized after psychostimulant treatment (Sobel et al, 2010). Longitudinal measurements of cerebral cortex thickness indicated that ADHD-associated excess cortical thinning during adolescence could be moderated by psychostimulant treatment (Shaw et al, 2009). A recent voxel-based meta-analysis that included pediatric and adult samples further confirmed that ADHD patients had a development-related deficit in local gray matter volume, and that this may be resolved with stimulant medication (Nakao et al, 2011).

However, patient studies, such as described above, are typically hampered by the heterogeneity of the population sample, with mixed treatment histories and comorbidity of psychiatric disorders, due to their retrospective design. Moreover, lack of appropriate controls means that the effects of long-term psychostimulant exposure during normal brain development remain elusive. Animal models may therefore afford a well-controlled experimental setup to disentangle the effects of neuropathology and chronic psychostimulant treatment on the developing brain. Studies in rats have shown that early exposure to methylphenidate can affect midbrain dopaminergic neurons (Brandon et al, 2003), cause profound reductions in striatal dopamine transporter levels (Moll et al, 2001), but could also prevent methamphetamine-triggered suppression of dopaminergic innervation of the prefrontal cortex and amygdala (Grund et al, 2007). However, it remains unclear to what extent long-term exposure of the normally developing adolescent brain to methylphenidate-in the absence of ADHDaffects gross tissue morphology and function as observed in patient studies.

Therefore, our aim was to study the effects of chronic stimulant treatment on the healthy brain in a wellcontrolled experimental setting. In a first attempt to evaluate the differential effects of chronic psychostimulant medication on normal brain maturation, we treated normally developing adolescent and adult rats chronically with oral methylphenidate and applied high-resolution magnetic resonance imaging (MRI) to measure cortical thickness, gray matter and white matter tissue volumes, and diffusion tensor imaging (DTI)-based fractional anisotropy of water diffusion to assess white matter structural integrity. In addition, we used two in vivo functional MRI (fMRI) paradigms to study the effects of methylphenidate exposure on functional brain networks. Resting-state fMRI was used to measure baseline functional connectivity. As methylphenidate acts on the dopamine system by targeting the dopamine transporter (Volkow et al, 1998), we performed pharmacological MRI following a low-dose intravenous D-amphetamine injection that stimulates dopamine efflux, to assess whether long-term treatment affected dopaminemediated activation responses. Finally, myelin content was quantified with immunohistochemistry.

In line with previous clinical and experimental literature, we therefore hypothesized that methylphenidate treatment during adolescence, but not during adulthood, would stimulate white matter, striatal and frontal cortical development, resulting in more adult-like values, in particular larger white matter tissue volumes and increased DTI-based fractional anisotropy along white matter tracts.

\section{MATERIALS AND METHODS}

\section{Animals}

All experiments were approved by the Committee for Animal Experiments of the University Medical Center Utrecht, The Netherlands (2010.I.03.044), and all efforts were made to minimize animal suffering and to reduce the number of animals used.

The studies were conducted in male Wistar rats (Harlan, Horst, The Netherlands) from pre-adolescence throughout adolescence, or during adulthood. Adolescence in rats can be broadly defined as the period between post-natal day (P)28 and P60 (Brenhouse and Andersen, 2011; McCutcheon and Marinelli, 2009; Spear, 2000). Animals were treated with 0 (vehicle) or $5 \mathrm{mg} / \mathrm{kg}$ methylphenidate $\mathrm{HCl}(\mathrm{MPH})$ (oral gavage, dissolved in $0.9 \%$ saline solution, 1.25 or $2.5 \mathrm{mg} / \mathrm{ml}$ for adolescent and adult rats, respectively), daily for 21 days from P25 (adolescent) or P65 \pm 4 (adult), followed by a 1-week washout period. Sixty-four animals were included ( $n=16$ per group). Body weight was measured daily during treatment, and following the washout period. The methylphenidate dosing regimen used in the present study was chosen to mimic clinically therapeutic doses and route of administration in rats (Gerasimov et al, 2000; Grund et al, 2007).

\section{In vivo $\mathrm{MRI}$}

In vivo brain status was assessed on a $4.7 \mathrm{~T}$ horizontal bore MR system (Agilent, Santa Clara, CA). During MRI, anesthesia was maintained with $60 \mathrm{mg} / \mathrm{kg} / \mathrm{h}$ propofol i.v. Animals were ventilated with a mixture of $\mathrm{O}_{2}$ /air $(1: 7)$. Blood oxygen saturation, heart rate, and end-tidal $\mathrm{CO}_{2}$ were monitored, and body temperature was maintained at $37.0 \pm 0.5^{\circ} \mathrm{C}$.

Anatomical images were obtained with a $\mathrm{T}_{2}$-weighted $3 \mathrm{D}$ gradient-echo (GE) sequence (repetition time (TR)/echo time $(\mathrm{TE})=6 / 2.25 \mathrm{~ms} ; 40^{\circ}$ flip angle; $0.23 \times 0.31 \times 0.31 \mathrm{~mm}^{3}$ voxels). $10 \mathrm{~min}$ of resting-state fMRI was performed with a $\mathrm{T}_{2}{ }^{*}$-weighted single-shot GE-EPI sequence $(\mathrm{TR} / \mathrm{TE}=600 /$ $25 \mathrm{~ms} ; 50^{\circ}$ flip angle; $0.5 \times 0.5 \mathrm{~mm}^{2}$ voxels; $13 \times 1.5 \mathrm{~mm}^{2}$ coronal slices; 1000 images). To assess whether long-term methylphenidate exposure induced sustained alterations to dopaminergic neuronal signaling, pharmacological MRI with a dopamine-releasing agent was performed using a four-shot GE-EPI sequence $\left(\mathrm{TR} / \mathrm{TE}=1000 / 25 \mathrm{~ms} ; 65^{\circ}\right.$ flip angle; $0.5 \times 0.5 \mathrm{~mm}^{2}$ voxels; $19 \times 1.0 \mathrm{~mm}^{2}$ coronal slices; 675 images) with $10 \mathrm{~min}$ baseline scanning, and $35 \mathrm{~min}$ scanning following intravenous injection of 0 (vehicle; $n=3$ per group) or $1 \mathrm{mg} / \mathrm{kg}$ ( $n=13$ per group) D-amphetamine in saline $(1 \mathrm{mg} / \mathrm{ml})$. At this dose, $\mathrm{D}$-amphetamine has been shown to induce robust hemodynamic activation responses (Schwarz et al, 2004) that correlate with extracellular dopamine levels (Ren et al, 2009).

Immediately after MRI, animals were euthanized with pentobarbital ( $300 \mathrm{mg} / \mathrm{kg}$, i.p.) and transcardially perfused with cold phosphate buffered saline (PBS) followed by $4 \%$ formaldehyde in PBS. After overnight post-fixation in the skull, the brains were removed and stored in PBS with sodium azide.

One animal died during transition to propofol, and four were excluded from the pharmacological MRI analysis 
because of physiological instability or complications during preparation.

\section{Postmortem MRI}

Dissected brains ( $n=12$ per group) were measured on a 9.4T horizontal bore MR system (Agilent). Brains were placed in a custom-made holder and immersed in a nonmagnetic oil. Anatomical images with an isotropic voxel size of $93.75 \mu \mathrm{m}$ were acquired (3D GE; TR/TE $=6.87 / 3.34 \mathrm{~ms}$; $15^{\circ}$ flip angle). DTI was performed to assess white matter status, using a diffusion-weighted eight-shot spin-echo EPI sequence $\quad\left(\mathrm{TR} / \mathrm{TE}=2700 / 28 \mathrm{~ms} ; \quad 0.2 \times 0.2 \mathrm{~mm}^{2} \quad\right.$ voxels; $55 \times 0.2 \mathrm{~mm}^{2}$ transversal slices; $b=3174 \mathrm{~s} / \mathrm{mm}^{2}, \delta=5 \mathrm{~ms}$, $\Delta=11 \mathrm{~ms}$; two sets of 60 diffusion-weighted images in noncollinear directions, and six unweighted images $(b=0))$.

\section{Immunohistochemistry}

In a subset of the brains of experimental animals $(n=7-8$ per group) 2',3'-cyclic nucleotide $3^{\prime}$-phosphodiesterase (CNPase) immunohistochemistry was used to quantify myelin content in the corpus callosum, anterior commissure, and striatum (at 2.28, 1.32, 0.60, and $-0.60 \mathrm{~mm}$ relative to bregma).

Supplementary Materials and Methods provide detailed description of the sectioning and staining procedure, as well as the above described MRI acquisitions.

\section{Statistics}

Unless stated otherwise, data were statistically analyzed using linear model, ANOVA, or linear mixed-model analysis to test for effects of (i) age (P25-P65), (ii) treatment (vehicle-MPH), and (iii) and age-by-treatment interaction. Linear mixed-model analysis was performed in $R$ (www. r-project.org) using nlme, or lme4 and languageR packages. Following multiple non-independent tests, $p$-values were false discovery rate (FDR)-adjusted and voxel-based statistics were cluster-corrected. Minimum cluster sizes were computed within FSL FEAT, or obtained by Monte Carlo simulations implemented in AlphaSim (Ward, 2000).

\section{Data Analysis-In Vivo MRI}

Image pre-processing. Image intensity non-uniformity correction was performed (Sled et al, 1998), and brain masks were obtained using BET as implemented in FSL (Jenkinson et al, 2012). Using elastix (Klein et al, 2010), within-subject images were aligned and subsequently registered non-rigidly to an anatomical reference image. Resting-state fMRI pre-processing was performed as described before (van Meer et al, 2012). Pharmacological MRI images were corrected for subject motion using FSL FLIRT (Jenkinson et al, 2002), and spatially smoothed (1.0 mm Gaussian kernel).

Resting-state fMRI. Group-level independent component analysis (ICA) of resting-state fMRI data was performed using temporal concatenated spatial ICA as implemented in FSL MELODIC (Beckmann and Smith, 2005). Subject-level spatial maps and associated time courses were obtained with the dual-regression approach (Filippini et al, 2009). To verify that the results did not depend on the a priori defined number of components (20), ICA was replicated with 30 and 40 components. Subsequent analyses were confined to components that represented three distinct and reproducibly observed networks (see Results). For each reported component, the within-subject intranetwork functional connectivity was calculated as the average Fisher $z^{\prime}$-transformed partial correlation coefficient of its subjectlevel time-course with the signal in all voxels identified by the group-level spatial map (thresholded at $Z>1.6$ ). In a single linear mixed-model analysis, the estimates and associated $t$ - and $p$-values for age, treatment, and age-bytreatment interaction effects were obtained for each of the three components. Component was fitted as an additional fixed effect, subject as a random effect, and a general correlation structure was used to model within-subject dependencies.

Pharmacological MRI. Subject-level analysis of pharmacological MRI images was performed within FSL FEAT, using a typical D-amphetamine response curve estimated from all subjects' pharmacological MRI data, the response curve's first-order time derivative, and motion correction parameters. Group-level tensorial ICA as implemented in FSL MELODIC (Beckmann and Smith, 2005) was used to extract the response curve, after temporal smoothing with a 15-point Hamming filter using AFNI (Cox, 1996). Total activation in the dorsomedial prefrontal cortex and striatum was calculated as the integral of the relative blood oxygen level-dependent (BOLD) signal change after D-amphetamine injection, and was statistically tested with a linear model analysis. Voxel-wise second-level mixed effects analysis was performed using FLAME as implemented in FSL FEAT. Normalized $(Z)$ statistic maps were thresholded according to $Z>2.3$ and cluster-corrected $(p<0.01)$ to detect voxels, which exhibited significant effects of age, treatment, or ageby-treatment interaction.

\section{Data Analysis-Postmortem MRI}

Morphometry template construction. A template image for morphometry analysis was iteratively refined by applying (i) non-rigid registration of subject images to the current template using ANTS (Avants et al, 2011), (ii) averaging of the affine transformations (Woods, 2003) and deformation fields, (iii) resampling subject images to the new template space, and (iv) averaging the resampled images to construct the updated template.

Diffusion tensor imaging. Analysis of DTI data was performed using Diffusion Toolkit (Wang et al, 2007) to obtain parameter maps based on voxel-wise estimates of the diffusion tensor, including fractional anisotropy and mean diffusivity. Images without diffusion weighting were nonrigidly registered to maximize alignment across subjects. A tract-based spatial statistics analysis was performed, which alleviates the effects of small registration inaccuracies and reduces the number of statistical tests to a study-specific white matter skeleton (Smith et al, 2006). Briefly, a common white matter skeleton was computed, and for each voxel on 
the skeleton fractional anisotropy values were tested for effects of age, treatment, and age-by-treatment interaction using linear model analysis.

In addition, fractional anisotropy values were measured in the same three regions and four planes as defined for the immunohistochemistry analysis (see above). A single linear mixed effects model was fitted to obtain estimates and associated $t$ - and $p$-values of age, treatment, and age-bytreatment interaction effects for each region of interest. A general correlation structure explicitly modeled withinsubject dependencies between measurements across adjacent slices.

Total tissue volumes. Major white matter tracts, cerebrospinal fluid, the cerebral cortex, and other cerebral gray matter tissue were outlined on the template image for ANOVA testing for effects of age, treatment, and age-bytreatment interaction on gross cerebral tissue volumes. In addition, tests were performed for the striatum (outlined between 2.28 and $-1.08 \mathrm{~mm}$ from bregma) and for the anterior cingulate cortex (between 2.76 and $-0.60 \mathrm{~mm}$ from bregma).

Deformation-based morphometry. The transformations that were obtained during template construction describe the local tissue volume changes that are needed to match the template, in addition to global size and shape as accommodated with an affine transformation. At the voxel level, volume expansion or compression was quantified by the determinant of a transformation's Jacobian matrix. Local cerebral gray and white matter tissue volumes were then tested in a voxel-wise deformation-based morphometry analysis (Ashburner and Friston, 2000), using the logtransformed determinant values and with total brain volume as a covariate, with a linear model design.

Cortical thickness. Measurements of the local thickness of the cerebral cortex were obtained as described in Lerch $e t$ al (2008). Briefly, the cerebral cortex was manually outlined on the template image and subsequently mapped to individual subjects. Cortical thickness was measured at 3000 vertices per hemisphere on a mid-cortical surface template. Statistical tests were run for each vertex using a linear model design to estimate effects of age, treatment, and ageby-treatment interaction. In addition, the mean effects over vertices comprising total hemispheric surface, motor, sensorimotor, and anterior cingulate cortex were analyzed using a linear model design.

\section{Data Analysis-Immunohistochemistry}

Images were acquired with a Zeiss microscope (Carl Zeiss Microscopy GmbH, Jena, Germany) using Neurolucida software (MBF Bioscience, Williston, VT) at $\times 2.5$ magnification (gain 0.0 ; offset -0.1 ; exposure time $55 \mathrm{~ms}$ ). Analysis was performed in ImageJ (National Institutes of Health, Bethesda, MD) to quantify immunoreactivity, and striatal nerve bundles using the particle analysis function (Papadopulos et al, 2007). The optical density measurements were corrected for lighting and background intensity, which was calculated as the average intensity in four fixed-size regions of interest that were placed in the caudate-putamen but outside the striatal nerve bundles. Correlations between measurements in regions across adjacent slices of the same subject were explicitly modeled in the linear mixed-model analysis, similar to the analysis of DTI-based fractional anisotropy (see above).

\section{RESULTS}

\section{Spontaneous and D-Amphetamine-Induced Neural Activation Responses}

Resting-state fMRI. Group-level spatial ICA of restingstate fMRI data consistently identified three networks (Figure 1a), which largely encompassed (i) the bilateral anterior cingulate and medial prefrontal cortices, (ii) the bilateral hippocampi, and (iii) the bilateral striata. Methylphenidate-treated animals showed a small but significant reduction on intranetwork functional connectivity (Figure $1 \mathrm{~b}$ ) in the bilateral anterior cingulate cortices $(t=-2.03, p<0.05)$. The bilateral striatum showed an effect of age, with a significantly larger intranetwork functional connectivity in adult animals $(t=4.06, p=0.001)$.

Pharmacological MRI. Figure 1c shows the D-amphetamine signal model and its first derivative. Second-level mixed effects analysis of pharmacological MRI data revealed no significant effects of treatment or age-by-treatment interaction. A significant age effect $(Z>2.3$, clustercorrected with $p<0.01$ ) was observed, with a stronger D-amphetamine-induced activation response in the dorsal striatum of adult animals as compared with adolescent animals (Figure 1d).

The integral of the BOLD signal time-course upon Damphetamine injection, relative to baseline, was calculated for the bilateral striata (P25: vehicle $=141 \pm 136 \%$ s, $\mathrm{MPH}=$ $153 \pm 59 \%$ s; P65: vehicle $=286 \pm 145 \%$ s, $\mathrm{MPH}=338 \pm 99 \%$ s) and for the bilateral dorsomedial prefrontal cortices (P25: vehicle $=220 \pm 205 \%$ s, $\mathrm{MPH}=231 \pm 89 \%$ s; P65: vehicle $=$ $335 \pm 287 \%$ s, $\quad \mathrm{MPH}=349 \pm 191 \%$ s). Linear model tests revealed no significant effect of treatment nor age-bytreatment. A significant effect of age was observed in the striata $(t=3.16, p=0.003)$ but not in the dorsomedial prefrontal cortices $(t=1.62, p=0.11)$.

\section{Gray and White Matter Volumes}

Total tissue volumes. Total gray matter, white matter, and cerebrospinal fluid volumes showed no significant treatment or age-by-treatment effect (Table 1). A significant effect of age was found for white matter and gray matter tissue volumes, but not for cerebrospinal fluid. Compared with adolescents, adults had overall more white matter (95\% confidence interval, $\left.\mathrm{CI}_{95 \%}=0.19-0.49 \%, p<0.001\right)$ and less gray matter $\left(\mathrm{CI}_{95 \%}=0.33-0.81 \%, p<0.001\right)$ relative to total cerebral volume (Table 1). Overall cerebrospinal fluid volumes did not significantly vary with age or treatment.

Regional tissue volumes. An effect of age-by-treatment interaction was found in the striatum $(\mathrm{F}=5.56, p=0.02)$ due to a treatment-related $1.6 \%$ reduction in volume in adolescents (from $3.41 \pm 0.10 \%$ to $3.36 \pm 0.10 \%$ ), and a $2.8 \%$ 
a
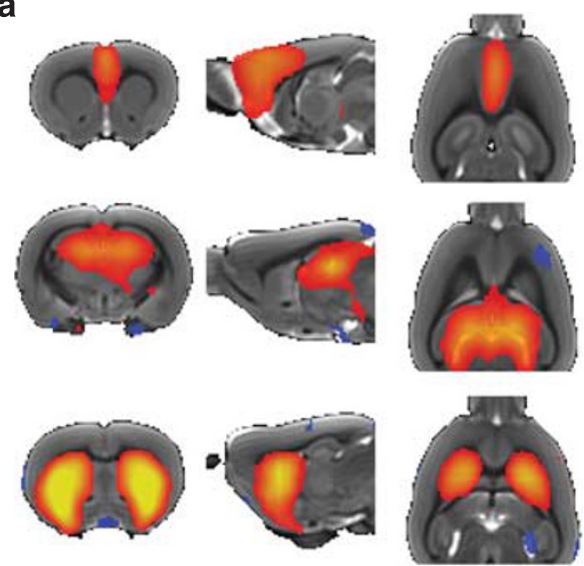

$-15$

$\begin{array}{lll}-2.3 & Z & 2.3\end{array}$

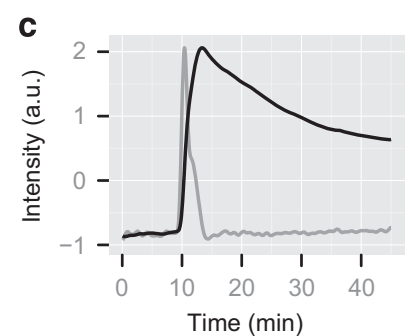

b

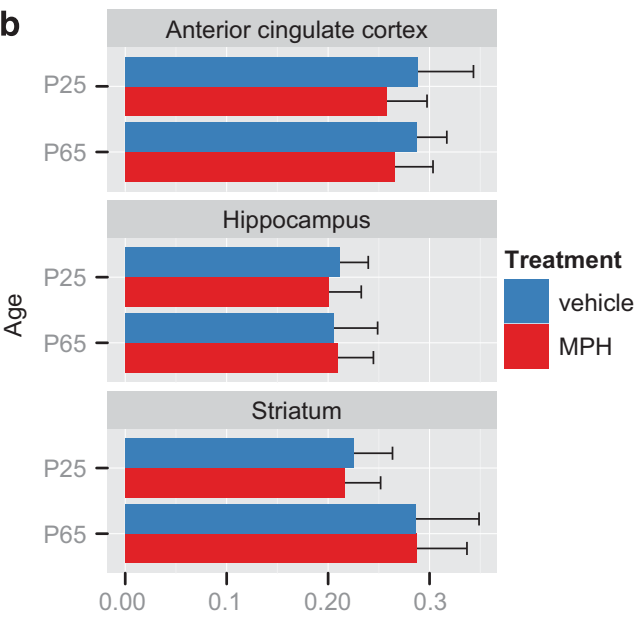

Average functional connectivity $\left(z^{\prime}\right)$

Figure I Spontaneous and D-amphetamine-induced neural activation responses. (a) Coronal, sagittal, and transversal slices of a template image overlaid with voxels belonging to three typical resting-state functional magnetic resonance imaging (fMRI) networks as obtained with group-level independent component analysis (ICA; $n=63$ ): the bilateral anterior cingulate and prefrontal cortices (first row), the bilateral dorsal hippocampi (second row), and the bilateral dorsal and ventral striata (third row). (b) Means and SD of intranetwork functional connectivity for each reported network (P25 + vehicle: $n=16$; P25 + MPH (methylphenidate HCl): $n=15 ;$ P65 + vehicle: $n=16 ;$ P65 + MPH: $n=16$ ). Linear mixed-model analysis revealed a significant effect of age for the striatal network, and a small effect of treatment for the anterior cingulate cortical network. (c) Typical blood oxygen level-dependent (BOLD)-response upon D-amphetamine injection (black curve) and its first-order time derivative (gray curve), obtained with group-level tensorial ICA of smoothed pharmacological MRI data $(n=48)$. (d) The typical response curve and its derivative (c) were used as regressors in a voxel-wise mixed-model fits effects analysis at the subject level, which revealed significant $(Z>2.3$, cluster-corrected with $p<0.01)$ effects of age-effects on the BOLD-response upon $D$ amphetamine injection (P25 + vehicle: $n=12 ; P 25+\mathrm{MPH}: n=12 ; \mathrm{P} 65+$ vehicle: $n=13 ; \mathrm{P} 65+\mathrm{MPH}: n=11$ ). The associated voxels are color-coded on coronal slices of a template image. An increased activation response was most strongly observed in the dorsal striata of adult animals (black arrowheads).

Table I Total and Regional Brain Tissue Volumes

\begin{tabular}{|c|c|c|c|c|c|c|c|}
\hline \multirow[t]{2}{*}{ Region of interest } & \multicolumn{4}{|c|}{ Mean \pm SD $\left(\mathbf{m m}^{3}\right)$} & \multicolumn{3}{|c|}{ Effects F-statistic ( $p$-value) } \\
\hline & \multicolumn{2}{|c|}{ Adolescent (P25) } & \multicolumn{2}{|c|}{ Adult (P65) } & Age & Treatment & Age $\times$ treatment \\
\hline Cerebrum & $1473 \pm 108$ & $1455 \pm 57$ & $1480 \pm 113$ & $1497 \pm 112$ & $0.03(0.87)$ & $0.18(0.67)$ & $0.36(0.55)$ \\
\hline Gray matter & $88.8 \pm 0.3 \%$ & $89.0 \pm 0.4 \%$ & $88.3 \pm 0.6 \%$ & $88.4 \pm 0.4 \%$ & $9.51(<0.01)^{*}$ & $1.16(0.29)$ & $0.16(0.69)$ \\
\hline Cerebrospinal fluid & $2.66 \pm 0.37 \%$ & $2.62 \pm 0.28 \%$ & $2.83 \pm 0.45 \%$ & $2.91 \pm 0.44 \%$ & $1.15(0.29)$ & $0.06(0.8 I)$ & $0.27(0.61)$ \\
\hline Striatum & $3.41 \pm 0.10 \%$ & $3.36 \pm 0.10 \%$ & $3.38 \pm 0.15 \%$ & $3.48 \pm 0.09 \%$ & $0.40(0.53)$ & $1.48(0.23)$ & $5.55(0.02)^{*}$ \\
\hline Anterior cingulate cortex & $2.95 \pm 0.15 \%$ & $2.96 \pm 0.08 \%$ & $2.79 \pm 0.11 \%$ & $2.80 \pm 0.16 \%$ & $9.11(<0.01)^{*}$ & $0.05(0.82)$ & $0.01(0.94)$ \\
\hline
\end{tabular}

Means and standard deviations (SD) of total cerebral tissue volume (absolute), and volume relative to total cerebral tissue for gray matter, white matter, cerebrospinal fluid, striatum, and anterior cingulate cortex. Statistical testing of the absolute or relative tissue volumes across groups was performed using separate ANOVA tests, yielding F-statistics and corresponding $p$-values for the effects of age, treatment, and the age-by-treatment interaction. ${ }^{*} p<0.05$.

increase in adults (from $3.38 \pm 0.15 \%$ to $3.48 \pm 0.09 \%$ ). An age-effect, but no treatment or age-by-treatment effect, was observed in the anterior cingulate cortex, which was larger in adolescent rats $\left(\mathrm{CI}_{95 \%}=0.09-0.24 \%, p<0.001\right)$.
Deformation-based morphometry. Deformation-based morphometry revealed small effects of age-by-treatment (Figure 2a) mostly caudally between cerebrum and cerebellum, around the superior colliculus, with a 
a

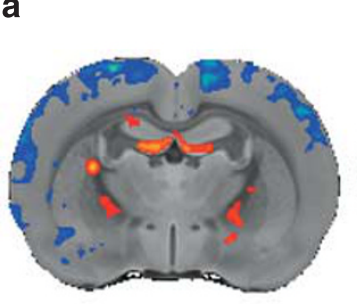

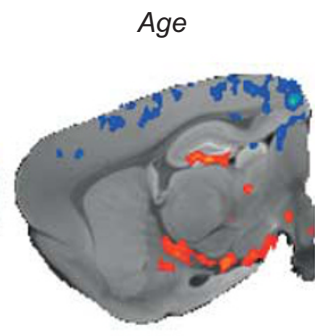

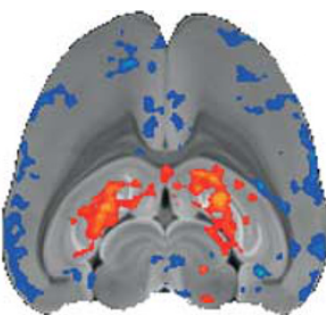

Age $\times$ Treatment
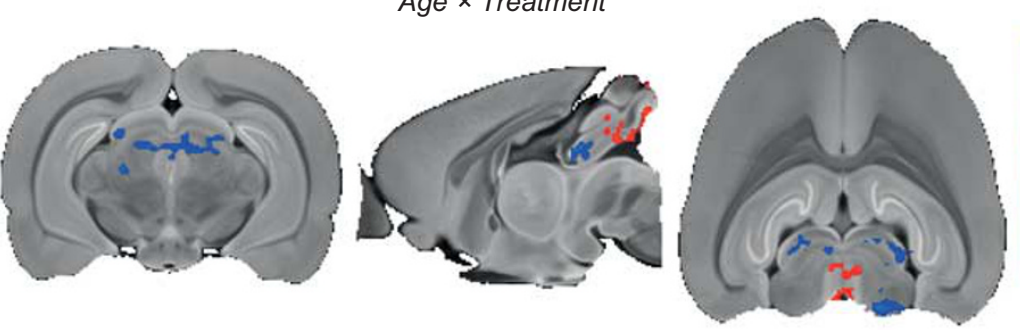

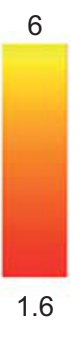

Z
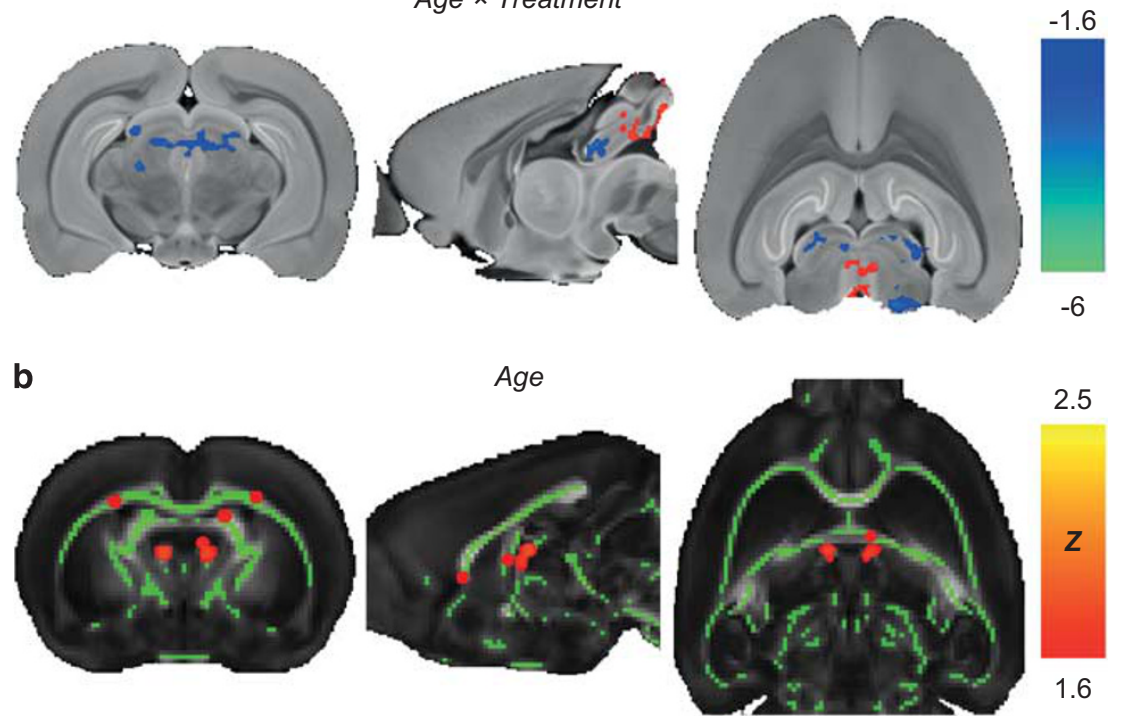

2.5

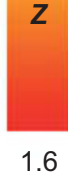

Figure 2 Deformation-based morphometry and tract-based spatial statistics. Deformation-based morphometry analysis revealed widespread significant effects of age on relative regional tissue volumes and a small age-by-treatment interaction effect $(n=48)$. (a) Shows cluster-corrected (cluster- $p<0.0 \mathrm{I})$ statistical Z-maps of the effects of age (top row; $p<0.05$ ) and age-by-treatment interaction (bottom row; $p<0.05$ ) overlaid on a coronal, sagittal, and transversal slice of a template image. Voxel-based analysis of fractional anisotropy (b; coronal, sagittal, and transversal slices of a template fractional anisotropy image) was restricted to a white matter skeleton (green; fractional anistropy $>0.3$ ) using tract-based spatial statistics. Linear model analysis revealed significant age effects (false discovery rate (FDR)-corrected $p<0.05$; voxels with significant differences were smoothed for visualization purposes). Both morphometry and fractional anisotropy analyses did not reveal a significant effect of treatment.

treatment-related volume decrease of $3.9 \%$ in adolescents, and an increase of $1.1 \%$ in adults.

A significant age effect was found, with adults displaying relatively smaller cortical tissue volumes than adolescents. Conversely, relative subcortical (eg, hippocampus) tissue volumes were larger in adults. On average, significant adultincreased voxels reflected a tissue volume increase of $26.0 \pm 10.2 \%$, whereas adult-decreased voxels reflected a decrease of $15.9 \pm 5.1 \%$.

\section{White Matter Integrity}

Fractional anisotropy. Tract-based spatial statistics revealed no effects of treatment or age-by-treatment. Age effects were identified in white matter voxels where fractional anisotropy was significantly increased in adult animals (Figure $2 b$ ).

In addition, fractional anisotropy was measured in the corpus callosum, anterior commissure, and striatum (Figure 3, left panel; and Supplementary Table S1). Linear mixed-model analysis revealed age $(t=3.22, p=0.001)$, treatment $(t=3.12$, $p=0.002)$, and age-by-treatment $(t=-2.30, p=0.02)$ effects on fractional anisotropy in the corpus callosum region. Upon methylphenidate treatment, fractional anisotropy in the corpus callosum was 9.2\% higher in adolescents and $0.3 \%$ lower in adults as compared with vehicle-treated rats. An ageeffect $(t=1.99, p=0.05)$ was observed in the anterior commissure, with fractional anisotropy $3.6 \%$ higher in adult as compared with adolescent rats.

Immunohistochemistry. The immunohistochemical staining with anti-CNPase was performed to assess brain myelination status (Figure 3, right panel; and Supplementary Table S1). Corpus callosum and anterior commissure myelination was not significantly different across groups, but the amount of CNPase immunoreactivity in the striatal region showed a significant effect of treatment $(t=-2.06$, $p=0.04)$ and age-by-treatment $(t=2.13, p=0.03)$, indicative of a decrease in myelin content upon treatment in adolescents $(-9.7 \%)$ but not in adults $(+3.1 \%)$, and a trend of an age-effect $(t=-1.91, p=0.06)$.

\section{Cortical Thickness}

Treatment, nor age-by-treatment interaction, had a significant effect on cortical thickness. Overall, adult animals had slightly thinner cortices than adolescent animals (Figure 4). Among vehicle-treated animals, the average thickness over the cerebral cortex was $1.52 \pm 0.50 \mathrm{~mm}$ in adolescents, and $1.49 \pm 0.48 \mathrm{~mm}$ in adults. The average 

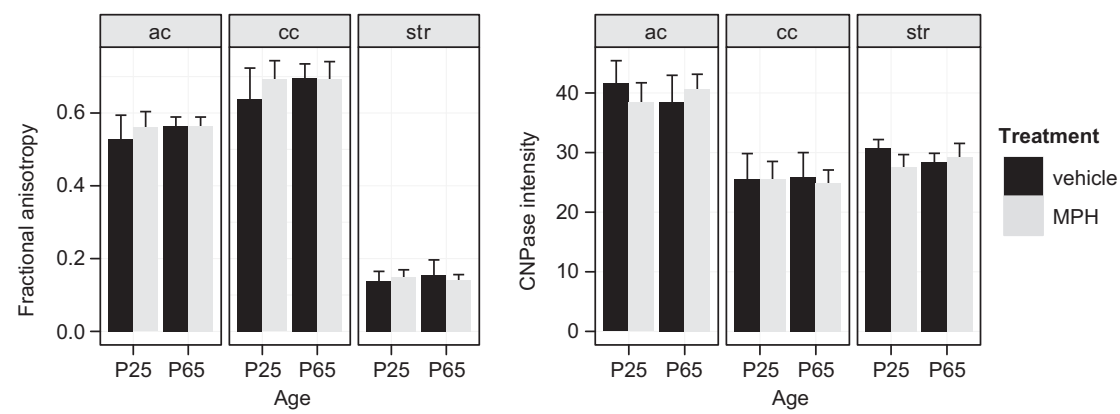

Figure 3 Diffusion tensor imaging (DTI) fractional anisotropy and 2', $3^{\prime}$-cyclic nucleotide $3^{\prime}$-phosphodiesterase (CNPase) myelin staining. Effects of age and treatment on white matter integrity were studied in three regions of interest (ac: anterior commissure; cc: corpus callosum; str: striatum) with DTI-based measurements of fractional anisotropy, which is a value between zero and one that expresses the degree of diffusion anisotropy (left panel; P25 + vehicle: $n=12 ; \mathrm{P} 25+\mathrm{MPH}$ (methylphenidate $\mathrm{HCl}): n=12 ; \mathrm{P} 65+$ vehicle: $n=12 ; \mathrm{P} 65+\mathrm{MPH}: n=12$ ), and immunohistochemical staining of CNPase expressed as background-corrected intensity values (right panel; P25 + vehicle: $n=7 ; \mathrm{P} 25+\mathrm{MPH}: n=8 ; \mathrm{P} 65+$ vehicle: $n=8 ; \mathrm{P} 65+\mathrm{MPH}: n=8$ ). Bar graphs display mean and SD across subjects and corrected for differences across the four slice locations (at 2.28, I.32, 0.6, and - $0.6 \mathrm{~mm}$ from bregma) after linear mixedmodel analysis. Statistical tests revealed significant age, treatment, and age-by-treatment effects on DTI-based fractional anisotropy measured in the corpus callosum; an age-effect on fractional anisotropy in the anterior commissure; and a significant treatment and age-by-treatment effect on CNPase intensity in the striatal region.
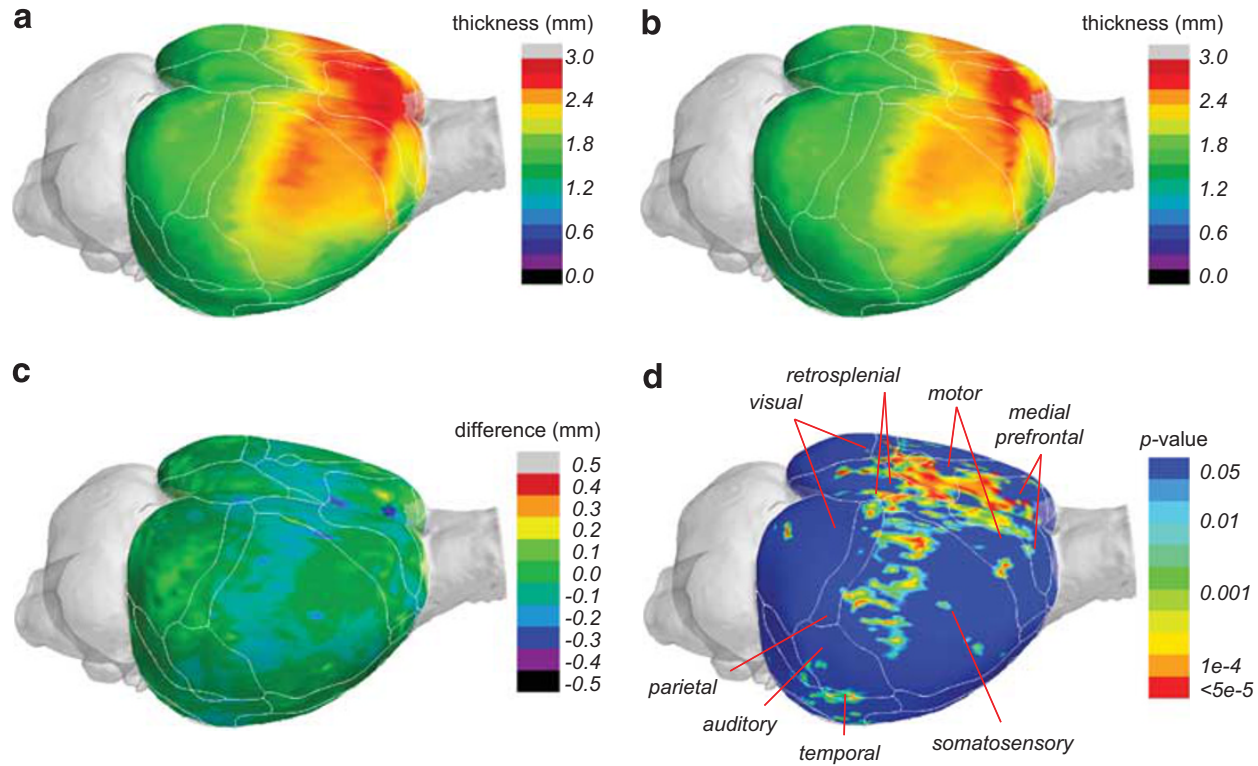

Figure 4 Cortical thickness. Cortical thickness was measured in all four groups, and tested for age and treatment effects at each point on the cortical surface with a linear model analysis $(n=48)$. (a, b) Show group-level estimates of the thickness projected on the cortical surface for vehicle-treated adolescent (a) and adult (b) animals. The mean difference between vehicle-treated adolescents and adults is projected in (c). A significant effect of age (false discovery rate (FDR)-corrected $p<0.05$ ) was found across the cerebral cortex, most extensively in medial prefrontal, motor, somatosensory, mid-cingulate, retrosplenial, and temporal cortices (d).

decrease in cortical thickness between the two ages was $0.035 \pm 0.063 \mathrm{~mm}$.

Region-of-interest analysis revealed significant decrease of cortical thickness with age across all vertices $(t=-2.12$, $p=0.04)$, and specifically for vertices in the motor cortex $(t=-3.48, \quad p=0.001)$, somatosensory cortex $(t=2.22$, $p=0.03)$, and anterior cingulate cortex $(t=-3.40, p=0.001)$.

\section{Body Weight}

We observed that methylphenidate-treated animals did not gain as much body weight as control animals did. Therefore, subject-level estimates of body weight gain ( $\mathrm{kg} /$ day) were calculated using linear regression. Methylphenidate treatment was found to significantly reduce weight development by $0.4 \times 10^{-3} \mathrm{~kg} /$ day $(\mathrm{F}=4.19, p=0.045)$. Treatment effects were similar for adolescents and adults, and did not result in significantly different body weights after the washout period $(\mathrm{F}=1.81, p=0.18)$. Body weight of adolescent animals increased $7.5 \pm 0.6 \cdot 10^{-3} \mathrm{~kg} /$ day, while adults showed weight gains of $3.3 \pm 0.9 \cdot 10^{-3} \mathrm{~kg} /$ day $(\mathrm{F}=511.5$, $p<0.001)$.

\section{DISCUSSION}

In the present study, we measured the differential effects of adolescent and adult methylphenidate treatment on functional and structural characteristics of the rat brain. We detected small effects of methylphenidate treatment, which frequently depended on age of treatment onset. Upon 
methylphenidate treatment, anterior cingulate cortical intranetwork functional connectivity was decreased and adolescent rats displayed decreased striatal myelination, striatal volumes, and increased fractional anisotropy in the corpus callosum. Morphology and volume of cortical and other subcortical tissues, as well as total white matter volume, were mostly unaffected by treatment. Furthermore, methylphenidate suppressed body weight gain in both adults and adolescents. In addition, many age-related features were significantly different between adult and adolescent rats, regardless of drug exposure.

\section{Methylphenidate Affects Striatal Morphology and Frontal Connectivity}

In the striatal region, we observed an age-by-treatment effect for total volume as well as for CNPase immunoreactivity: after treatment a reduction was observed only in adolescent rats, and not in adult rats. Treatment effects on white matter were observed for fractional anisotropy in the anterior part of the corpus callosum, which increased with both age and treatment. At the same time, functional connectivity in the anterior cingulate cortical network was consistently smaller in the treatment groups.

The effects of methylphenidate on striatal morphology echo clinical observations of medication-induced normalization of inward caudate nuclei deformations (Sobel et al, 2010). These findings are in line with a recent meta-analysis (Nakao et al, 2011) that indicated that in ADHD, and in terms of medication effects, the most prominent and replicable structural abnormalities occur in the basal ganglia.

The structural and functional frontostriatal alterations observed in this study may have resulted from methylphenidate's interference with the developing dopamine system. Corticostriatal afferents and nigrostriatal dopaminergic innervation converge on striatal spiny projection neurons (Gerfen, 2004), while methylphenidate preferentially and effectively blocks the dopamine transporter (Volkow et al, 1998). Binding studies have found 50\% reductions of adult dopamine transporter densities in the striatum following methylphenidate treatment in early adolescent, but not adult rats (Moll et al, 2001).

However, the functional relevance of the presently observed modest treatment effects on striatal morphology remains unclear. In any case, they were not accompanied by significant differences in spontaneous activity as measured with resting-state fMRI. Furthermore, pharmacological MRI did not provide evidence for methylphenidate-induced alterations to other parts of the dopaminergic system.

\section{Total Gray and White Matter Tissue Volumes are Mostly Unaffected by Treatment}

Small clusters of age-by-treatment effects on local cerebral tissue volumes were found caudally near the cerebellum and around the superior colliculus, while most cerebral gray matter tissue volumes were not significantly different between treatment and control groups. The reduced functional connectivity in the anterior cingulate cortical network was also not paralleled by macrostructural changes in gray matter organization, eg, cortical tissue volume or thickness measurements. Similarly, total white matter volume measurements did not reveal an effect of methylphenidate treatment.

In the clinical literature, stimulant medication also did not greatly affect gray matter volumes in ADHD patients (Castellanos et al, 2002). In contrast to our results, medication has been associated with normalization of lowered overall white matter volumes in ADHD patients in the order of $8.4-9.2 \%$ (Castellanos et al, 2002) and has been related to normalization of the volume of the right anterior cingulate cortex of children with ADHD (SemrudClikeman et al, 2006). Furthermore, children with ADHD who were off medication showed more rapid cortical thinning in dorsolateral prefrontal and motor regions than a typically developing cohort and children who received psychostimulants (Shaw et al, 2009).

\section{Limited Effects of Methylphenidate Exposure on Healthy Brains}

The absence of uniform treatment effects in the current observations could be attributed to differential effects of methylphenidate treatment on dopaminergic signaling in normal and pathological conditions. Clearly, healthy adolescents have not been included in clinical trials assessing methylphenidate's effects on brain function and structure for medical ethical reasons. In a previous study in gerbils, adolescent treatment with methylphenidate normalized dopaminergic fiber density in animals with methamphetamine-lesioned brains, but it did not affect control animals (Grund et al, 2007). On the other hand, other studies have also reported long-lasting changes in healthy animals, eg, reduced striatal dopamine transporter densities (Moll et al, 2001) and altered firing rates of midbrain dopaminergic neurons (Brandon et al, 2003). In line with this, behaviorally, pre-adolescent treatment with methylphenidate increased aversion to cocaine and reduced response to its rewarding properties in adult rats (Andersen et al, 2002), while long-term oral treatment during adolescence hardly affected measures of anxietylike behavior in otherwise healthy rats (Britton and Bethancourt, 2009). Furthermore, two recent studies have also reported no adverse effects of long-term psychostimulant treatment in young non-human primates on physical growth, motor and cognitive behavior, and dopaminerelated measures in relation to substance abuse (Gill et al, 2012; Soto et al, 2012).

In contrast to patient studies that report global or regionspecific stimulation of cortical neuroplasticity following methylphenidate treatment on otherwise overall delayed development trajectories (Shaw et al, 2009), our present study suggests that neurotrophic effects of methylphenidate on healthy rodent brains are much smaller. Although volume and myelination of the bilateral striata were differentially affected in adolescent and adult rats upon methylphenidate treatment, striatal functional activity seemed unaffected during resting conditions. Dopaminemediated activation responses were also unaffected by methylphenidate, which was assessed using pharmacological MRI with an injection of D-amphetamine. This psychostimulant drug principally targets dopamine and vesicular monoamine transporters (Fleckenstein et al, 2007), and depends on intact dopamine transporter function to cause 
pre-synaptic dopamine efflux (Jones et al, 1998). Behaviorally, functional deficits may only occur once a certain threshold is passed, eg, it is well known that parkinsonian signs do not occur before more than $50 \%$ of the dopamine terminals are degenerated (Fearnley and Lees, 1991).

We observed reduced development of body weight during methylphenidate treatment, which corresponds to earlier reports of reduced weight gain in rats (Gray et al, 2007). Similarly, the Multimodal Treatment of ADHD study has provided evidence for methylphenidate's potential growth suppression of weight and height (Faraone et al, 2008; Jensen et al, 2007).

\section{Age-Related Differences Between Adolescent and Adult Rats}

The effects related to age-differences at the time of measurement are likely to capture ongoing delayed neurodevelopmental processes occurring during early adulthood (Brenhouse and Andersen, 2011). For instance, humans attain peak cortical thickness region-dependently between 7 and 10.5 years (Shaw et al, 2007, 2008). This has been associated with pruning following early adolescent synaptogenesis, and myelination processes continuing well into adulthood (Brenhouse and Andersen, 2011). The increased $\mathrm{D}$-amphetamine response and intranetwork functional connectivity within the adult striatal network, as observed in our study, may partially reflect a continued development of dopaminergic pathways, with regard to midbrain dopaminergic neuron firing, and expression of striatal dopamine transporters and post-synaptic D1- and D2-like receptors (Chen et al, 2010; McCutcheon and Marinelli, 2009; Teicher et al, 1995).

\section{Assessment of White Matter Integrity}

The histological assessment of myelination in major interhemispheric fiber bundles and the striatum revealed complementary information to the diffusion-based measurement of tissue water fractional anisotropy in these regions. Although diffusion-based anisotropy measures may provide sensitive biomarkers of white matter integrity, many neuronal tissue characteristics, eg, axonal density and the degree of myelination, contribute to fractional anisotropy (Le Bihan, 2003). On the other hand, limited spatial resolution of the diffusion-weighted MR images relative to the dimensions and distribution of myelinated axons in the striatum may have left striatal myelination changes undetected with DTI.

\section{CONCLUSION}

In sum, many of the present observations concerned development-related differences between late adolescent and young adult rats. Nonetheless, methylphenidate treatment differentially affected both striatal volume and myelination in adolescent and adult rats, which corroborates evidence that most prominent medication effects in ADHD patients occur in the basal ganglia (Nakao et al, 2011). However, we were not able to replicate other reported clinical findings of stimulation of white matter volume or (large) increases in cortical thickness. Still, our measures of striatal function corroborate experimental studies that revealed no negative behavioral effects of long-term psychostimulant treatment on healthy animals. Future experimental studies in animal models of ADHD need to identify whether the discrepancy between animal and clinical findings are related to absence of neuropathology in the present study, or that the stimulating effects of methylphenidate in clinical studies have been overrated. Evidently, only a randomized clinical trial with methylphenidate in young and adult ADHD patients can decisively answer what effects chronic stimulant treatment has on the human brain.

Increasing evidence suggests that the long-term effects of drug exposure on the developing brain are delayed and expressed during adulthood (Andersen and Navalta, 2004). The presently observed effects of methylphenidate on the rat striatum were modest, but opposite in adolescent and adult rats. As we do not yet know whether these effects are reversible or not, and whether they are related to functional deficits over a longer period of time, our study highlights the importance for further research on this topic in children and adolescents treated with methylphenidate. Our findings are also of relevance to an increasing group of children that is being treated with stimulants, but do not suffer from $\mathrm{ADHD}$, for instance to increase school performance (Lakhan and Kirchgessner, 2012) or because of misdiagnosis (Elder, 2010).

\section{FUNDING AND DISCLOSURE}

The grant providers had no further role in the design of the study, in the collection, analysis and interpretation of data, in the writing of the report, and in the decision to submit the paper for publication.

\section{ACKNOWLEDGEMENTS}

We thank Annette van der Toorn, Gerard van Vliet, and Wouter Mol for their technical assistance. This work has been financially supported by grants of the Academic Medical Center (Fellowship; LR) and Utrecht University (High Potential program; RMD). LR, PJL and GM were supported by Priomed. PJL is supported by the Hersenstichting Nederland.

\section{REFERENCES}

Andersen SL, Arvanitogiannis A, Pliakas AM, LeBlanc C, Carlezon WA (2002). Altered responsiveness to cocaine in rats exposed to methylphenidate during development. Nat Neurosci 5: 13-14.

Andersen SL, Navalta CP (2004). Altering the course of neurodevelopment: a framework for understanding the enduring effects of psychotropic drugs. Int J Dev Neurosci 22: 423-440.

Ashburner J, Friston KJ (2000). Voxel-based morphometry. The methods. NeuroImage 11: 805-821.

Ashtari M, Kumra S, Bhaskar SL, Clarke T, Thaden E, Cervellione $\mathrm{KL}$ et al (2005). Attention-deficit/hyperactivity disorder: a preliminary diffusion tensor imaging study. Biol Psychiatr 57: 448-455.

Avants BB, Tustison NJ, Song G, Cook PA, Klein A, Gee JC (2011). A reproducible evaluation of ANTs similarity metric performance in brain image registration. NeuroImage 54: 2033-2044. 
Beckmann CF, Smith SM (2005). Tensorial extensions of independent component analysis for multisubject FMRI analysis. NeuroImage 25: 294-311.

Brandon CL, Marinelli M, White FJ (2003). Adolescent exposure to methylphenidate alters the activity of rat midbrain dopamine neurons. Biol Psychiatr 54: 1338-1344.

Brenhouse HC, Andersen SL (2011). Developmental trajectories during adolescence in males and females: a cross-species understanding of underlying brain changes. Neurosci Biobehav Rev 35: 1687-1703.

Britton GB, Bethancourt JA (2009). Characterization of anxietyrelated responses in male rats following prolonged exposure to therapeutic doses of oral methylphenidate. Pharmacol Biochem Behav 93: 451-459.

Carmona S, Vilarroya O, Bielsa A, Trèmols V, Soliva JC, Rovira M et al (2005). Global and regional gray matter reductions in ADHD: a voxel-based morphometric study. Neurosci Lett 389: 88-93.

Castellanos FX, Lee PP, Sharp W, Jeffries NO, Greenstein DK, Clasen LS et al (2002). Developmental trajectories of brain volume abnormalities in children and adolescents with attention-deficit/hyperactivity disorder. JAMA 288: 1740-1748.

Chen YI, Choi J-K, Xu H, Ren J, Andersen SL, Jenkins BG (2010). Pharmacologic neuroimaging of the ontogeny of dopamine receptor function. Dev Neurosci 32: 125-138.

Cox RW (1996). AFNI: software for analysis and visualization of functional magnetic resonance neuroimages. Comput Biomed Res 29: 162-173.

Elder TE (2010). The importance of relative standards in ADHD diagnoses: evidence based on exact birth dates. J Health Econ 29: 641-656.

Faraone SV, Biederman J, Morley CP, Spencer TJ (2008). Effect of stimulants on height and weight: a review of the literature. J Am Acad Child Adolesc Psychiatr 47: 994-1009.

Fearnley JM, Lees AJ (1991). Ageing and Parkinson's disease: substantia nigra regional selectivity. Brain 114(Pt 5): 2283-2301.

Filippini N, MacIntosh BJ, Hough MG, Goodwin GM, Frisoni GB, Smith SM et al (2009). Distinct patterns of brain activity in young carriers of the APOE-epsilon4 allele. Proc Natl Acad Sci USA 106: 7209-7214.

Fleckenstein AE, Volz TJ, Riddle EL, Gibb JW, Hanson GR (2007). New insights into the mechanism of action of amphetamines. Annu Rev Pharmacol Toxicol 47: 681-698.

Gerasimov MR, Franceschi M, Volkow ND, Gifford A, Gatley SJ, Marsteller D et al (2000). Comparison between intraperitoneal and oral methylphenidate administration: a microdialysis and locomotor activity study. J Pharmacol Exp Ther 295: 51-57.

Gerfen CR (2004). Basal Ganglia. In: Paxinos G (ed) The Rat Nervous System. 3rd edn. Elsevier Academic Press: San Diego, CA, pp 455-508.

Giedd JN, Castellanos FX, Casey BJ, Kozuch P, King AC, Hamburger SD et al (1994). Quantitative morphology of the corpus callosum in attention deficit hyperactivity disorder. Am J Psychiatr 151: 665-669.

Gill KE, Pierre PJ, Daunais J, Bennett AJ, Martelle S, Gage HD et al (2012). Chronic treatment with extended release methylphenidate does not alter dopamine systems or increase vulnerability for cocaine self-administration: a study in nonhuman primates. Neuropsychopharmacology 37: 2555-2565.

Gray JD, Punsoni M, Tabori NE, Melton JT, Fanslow V, Ward MJ et al (2007). Methylphenidate administration to juvenile rats alters brain areas involved in cognition, motivated behaviors, appetite, and stress. J Neurosci 27: 7196-7207.

Grund T, Teuchert-Noodt G, Busche A, Neddens J, Brummelte S, Moll GH et al (2007). Administration of oral methylphenidate during adolescence prevents suppressive development of dopamine projections into prefrontal cortex and amygdala after an early pharmacological challenge in gerbils. Brain Res 1176: 124-132.
Jenkinson M, Bannister P, Brady M, Smith S (2002). Improved optimization for the robust and accurate linear registration and motion correction of brain images. NeuroImage 17: 825-841.

Jenkinson M, Beckmann CF, Behrens TEJ, Woolrich MW, Smith SM (2012). FSL. NeuroImage 62: 782-790.

Jensen PS, Arnold LE, Swanson JM, Vitiello B, Abikoff HB, Greenhill LL et al (2007). 3-Year follow-up of the NIMH MTA study. J Am Acad Child Adolesc Psychiatr 46: 989-1002.

Jones SR, Gainetdinov RR, Wightman RM, Caron MG (1998). Mechanisms of amphetamine action revealed in mice lacking the dopamine transporter. J Neurosci 18: 1979-1986.

Klein S, Staring M, Murphy K, Viergever MA, Pluim JPW (2010). Elastix: a toolbox for intensity-based medical image registration. IEEE Trans Med Imag 29: 196-205.

Lakhan SE, Kirchgessner A (2012). Prescription stimulants in individuals with and without attention deficit hyperactivity disorder: misuse, cognitive impact, and adverse effects. Brain Behav 2: 661-677.

Le Bihan D (2003). Looking into the functional architecture of the brain with diffusion MRI. Nat Rev Neurosci 4: 469-480.

Lerch JP, Carroll JB, Dorr A, Spring S, Evans AC, Hayden MR et al (2008). Cortical thickness measured from MRI in the YAC128 mouse model of Huntington's disease. NeuroImage 41: 243-251.

McCutcheon JE, Marinelli M (2009). Age matters. Eur J Neurosci 29: 997-1014.

Moll GH, Hause S, Rüther E, Rothenberger A, Huether G (2001). Early methylphenidate administration to young rats causes a persistent reduction in the density of striatal dopamine transporters. J Child Adolesc Psychopharmacol 11: 15-24.

Nakao T, Radua J, Rubia K, Mataix-Cols D (2011). Gray matter volume abnormalities in ADHD: voxel-based meta-analysis exploring the effects of age and stimulant medication. Am J Psychiatry 168: 1154-1163.

Papadopulos F, Spinelli M, Valente S, Foroni L, Orrico C, Alviano F et al (2007). Common tasks in microscopic and ultrastructural image analysis using ImageJ. Ultrastruct Pathol 31: 401-407.

Pastor PN, Reuben CA (2008). Diagnosed attention deficit hyperactivity disorder and learning disability: United States, 2004-2006. Vital Health Stat 10: 1-14.

Ren J, Xu H, Choi J-K, Jenkins BG, Chen YI (2009). Dopaminergic response to graded dopamine concentration elicited by four amphetamine doses. Synapse 63: 764-772.

Schwarz A, Gozzi A, Reese T, Bertani S, Crestan V, Hagan J et al (2004). Selective dopamine D(3) receptor antagonist SB-277011A potentiates phMRI response to acute amphetamine challenge in the rat brain. Synapse 54: 1-10.

Semrud-Clikeman M, Pliśzka SR, Lancaster J, Liotti M (2006). Volumetric MRI differences in treatment-naïve vs chronically treated children with ADHD. Neurology 67: 1023-1027.

Shaw P, Eckstrand K, Sharp W, Blumenthal J, Lerch JP, Greenstein D et al (2007). Attention-deficit/hyperactivity disorder is characterized by a delay in cortical maturation. Proc Natl Acad Sci USA 104: 19649-19654.

Shaw P, Kabani NJ, Lerch JP, Eckstrand K, Lenroot R, Gogtay N et al (2008). Neurodevelopmental trajectories of the human cerebral cortex. J Neurosci 28: 3586-3594.

Shaw P, Sharp WS, Morrison M, Eckstrand K, Greenstein DK, Clasen LS et al (2009). Psychostimulant treatment and the developing cortex in attention deficit hyperactivity disorder. Am J Psychiatr 166: 58-63.

Silk TJ, Vance A, Rinehart N, Bradshaw JL, Cunnington R (2009). White-matter abnormalities in attention deficit hyperactivity disorder: a diffusion tensor imaging study. Hum Brain Mapp 30: 2757-2765.

Sled JG, Zijdenbos P, Evans AC (1998). A nonparametric method for automatic correction of intensity nonuniformity in MRI data. IEEE Trans Med Imag 17: 87-97. 
Smith SM, Jenkinson M, Johansen-Berg H, Rueckert D, Nichols TE, Mackay CE et al (2006). Tract-based spatial statistics: voxelwise analysis of multi-subject diffusion data. NeuroImage 31: 1487-1505.

Sobel LJ, Bansal R, Maia TV, Sanchez J, Mazzone L, Durkin K et al (2010). Basal ganglia surface morphology and the effects of stimulant medications in youth with attention deficit hyperactivity disorder. Am J Psychiatr 167: 977-986.

Soto PL, Wilcox KM, Zhou Y, Ator NA, Riddle MA, Wong DF et al (2012). Long-term exposure to oral methylphenidate or dlamphetamine mixture in peri-adolescent rhesus monkeys: effects on physiology, behavior, and dopamine system development. Neuropsychopharmacology 37: 2566-2579.

Spear LP (2000). The adolescent brain and age-related behavioral manifestations. Neurosci Biobehav Rev 24: 417-463.

Teicher MH, Andersen SL, Hostetter JC (1995). Evidence for dopamine receptor pruning between adolescence and adulthood in striatum but not nucleus accumbens. Brain Res Dev Brain Res 89: $167-172$. van Meer MPA, Otte WM, van der Marel K, Nijboer $\mathrm{CH}$, Kavelaars A, Berkelbach van der Sprenkel JW et al (2012). Extent of bilateral neuronal network reorganization and functional recovery in relation to stroke severity. J Neurosci 32: 4495-4507.

Volkow ND, Wang GJ, Fowler JS, Gatley SJ, Logan J, Ding YS et al (1998). Dopamine transporter occupancies in the human brain induced by therapeutic doses of oral methylphenidate. Am J Psychiatr 155: 1325-1331.

Wang R, Benner T, Sorensen AG, Wedeen VJ (2007). Diffusion toolkit: a software package for diffusion imaging data processing and tractography. Proc Intl Soc Mag Reson Med 15: 3720

Ward BD (2000): Simultaneous inference for fMRI data http:// afni.nimh.nih.gov/afni/doc/manual/AlphaSim.

Woods RP (2003). Characterizing volume and surface deformations in an atlas framework. Theory, applications and implementation. NeuroImage 18: 769-788.

Supplementary Information accompanies the paper on the Neuropsychopharmacology website (http://www.nature.com/npp) 